\title{
Overflow of Muscle Activation by Proprioceptive Neuromuscular Facilitation in Standing Position: A Cross-Sectional Electromyographic Investigation in Healthy Individuals
}

Luciane Aparecida Pascucci Sande de Souza

Monica B. Curtarelli

Cyntia Rogean de Jesus Alves de Baptista

Valdeci Carlos Dionisio ( $\sim$ vcdionisio@gmail.com )

Universidade Federal de Uberlândia https://orcid.org/0000-0002-2532-1148

\section{Research}

Keywords: Electromyography, overflow, proprioceptive neuromuscular facilitation

Posted Date: May 14th, 2020

DOI: https://doi.org/10.21203/rs.3.rs-27511/v1

License: (c) (1) This work is licensed under a Creative Commons Attribution 4.0 International License. Read Full License 


\section{Abstract}

Background Although in several studies has been observed the principle of overflow of muscle activation, no one explored if this principle occurs in a standing position. This study aimed to investigate the occurrence and the condition of the overflow from upper limb migrating to inferior contralateral supported limb in standing position in healthy subjects.

Methods Cross-sectional study with one sample. Eleven healthy individuals with a mean age of $22( \pm 5.6)$ years were evaluated during 5 tasks applying the proprioceptive neuromuscular facilitation in left side 1) rest, 2) active diagonal primitive3) isotonic resistive diagonal primitive, 4) isometric resistive diagonal primitive, 5) active primitive diagonal with active extension of the right lower limb. The right side was monitored by electromyography activity of the tibial anterior, soleus, vastus medial oblique, rectus abdominis, tensor fasciae latae, gluteus maximus, gluteus medius, adductor longus muscles.

Results Results showed a difference between tasks for soleus muscle $(p<0.001)$, with higher clinical relevance $(d=0.87)$, and the task with diagonal primitive against isotonic resistance produced more electromyography activity $(p<0.001)$. For all muscles there was a clinical relevance $(d>0.55)$.

Conclusion The approach used in this study could improve the condition of distal muscles, mainly soleus, facilitating the acquisition of a standing position as soon as possible.

\section{Introduction}

Overflow of muscle activation or irradiation is the propagation of synergic muscle coactivation proceeding from a resisted movement [1]. The overflow begins in the stronger muscle groups and can reach any segments from proprioceptive inputs [2].

In healthy subjects, the irradiation occurred from trunk flexion, increasing dorsiflexion in the right, and left lower limbs [3]. Trunk extension increased plantar flexion in both lower limbs. Also, it was observed that the irradiation occurred from the left lower limb to the right lower limb, measured by the increase in tibialis anterior activity [4]. So, the contralateral muscular activity in the non-exercised limb is the result of the irradiation process.

In addition, another study showed that force irradiation during upper limb diagonal exercises is affected by diagonal direction, contraction intensity, and gender when performed by healthy participants [5]. Our results in a previous article [6] match with some of these findings. The position to elicit muscle response at a distance, ipsilateral, and contralateral to the stimulus. We also observed higher contralateral muscle recruitment for trunk and shoulder muscles during lower limb isometric diagonal compared to movements in other conditions.

However, no one of these previous studies investigated this principle in a standing position, which is very functional in the rehabilitation process. Here, we hypothesized that distinct electromyographic activity on lower limb muscles would be observed during different tasks applying the proprioceptive neuromuscular 
facilitation (PNF) in the contralateral upper limb in standing position. Also, we hypothesized that muscle recruitment would be superior during diagonal against maximum isometric resistance, and would be different between the muscles. Therefore, this study aimed to investigate the occurrence and the condition of the overflow from upper limb migrating to inferior contralateral supported limb muscles in standing position. This study can be the first step in the knowledge of the overflow principle in stand position.

\section{Methods}

\section{Participants}

This is a cross-sectional study with one sample in different tasks using proprioceptive neuromuscular facilitation by the primitive diagonal for the upper limb. The Research Ethics Committee of the University approved this study (no. 1647/2010), and it was conducted on a motor control laboratory at the same University. The convenience sample recruited from university community, were eleven healthy individuals (5 females) with an average age of $22( \pm 5.6)$ years. The exclusion criteria were the presence of any musculoskeletal or neuromuscular disorders.

\section{Experimental set-up and data processing}

The tests were performed in the following position: partially seated at a height-adjustable table, keeping the left lower limb with hip and knee flexed at $90^{\circ}$. The right lower limb was positioned resting on the floor with the knee flexed at $15^{\circ}$ and hip in the neutral position.

Each subject was asked to perform 5 different tasks: 1) to remain at rest in the above position (R); 2$)$ perform the diagonal primitive (DP) of the left upper limb in the main pattern, with flexion, abduction and external rotation of the shoulder, elbow extension and total extension of the wrist and fingers [7]; 3) perform the same diagonal against isotonic resistance (DIR), where the therapist resists the patient's active movement through the entire range of motion (concentric contraction) of the same limb; 4) perform the same diagonal against maximum isometric resistance (DISO) in the $90^{\circ}$ range of the shoulder, total elbow extension and neutral wrist of the same limb; 5) perform a diagonal of the left upper limb and, concomitantly, perform an active extension of the right lower limb that was flexed at $15^{\circ}$ (DEXT).

We recorded the EMG activity of the tibialis anterior (TA), soleus (SO), vastus medial oblique (VMO), rectus abdominis (RA), tensor fasciae latae (TFL), gluteus maximus (GMX), gluteus medius (GMED), and adductor longus (AD) muscles using an EMG DelSYS electromyograph (model DE2.2L) with surface bipolar electrodes on the right side, with a total gain of 2000 and within a bandpass of $20-450 \mathrm{~Hz}$. The input impedance and CMRR of the EMG were, respectively, >1015 X/0.2 pF and $92 \mathrm{~dB}$ (typical). Positioning was done, according to [8]. An inter-electrode distance of $2 \mathrm{~cm}$ was kept constant. All EMG data were digitized at $1000 \mathrm{frames/s}$ using Optotrak software and a synchronization unit. 
The EMG data were processed offline using Matlab software (Math Works Inc., version 6.0). The EMG signals were rectified, filtered (low-pass at $20 \mathrm{~Hz}$ using a second-order Butterworth filter), and normalized to the averaged EMG signal recorded for the tested muscle during maximum voluntary isometric contraction (MVIC). The averaged EMG of the MVIC was calculated within the 500-1000 ms interval from the beginning of the isometric contraction.

\section{Statistical analysis}

To calculate the sample size was used the F tests family, ANOVA: repeated measures, within factors, effect size $f=0.50$, a error probability $=0.051-\beta$ error probability $=0.95$ for one group. It was determinate that the sample size should be at least 10 participants with power 0.95 . The data normality was tested and confirmed by the Kolmogorov-Smirnov test. We used a repeated-measures ANOVA test for each muscle in different tasks that were applied [muscle $x$ (5 tasks)]. The pair-wise comparisons with Bonferroni's correction were used to verify the main effects. All tests were performed in IBM@ SPSS $\odot$ (version 22.0.0.0), and we used for statistical significance level at 0.05 for all comparisons. Also, the clinical relevance of the findings was assessed using Cohen's test. According to Cohen [9], $d<0.50$ indicate small effects, $0.50 \leq d<0.80$ indicate medium effects, and $d \geq 0.80$ indicate large effects .

\section{Results}

The repeated measures ANOVA test reveals that TFL and SO muscles showed statistical difference between the tasks proposed (Table 1). The pair-wise comparison showed that for TFL, there was no statistical difference in all comparisons $(p>0.279)$. For SO muscle there was statistical difference in $\mathrm{R}$ vs $\operatorname{DIR}(p<0.001)$, R vs DISO ( $=0.001)$, R vs DEXT $(p=0.000)$, DP vs DIR $(p<0.003)$, DP vs DEXT $(p<$ $0.004)$ comparisons. However, for R vs DP $(p=0.096)$, DP vs DISO $(p=0.271)$, DIR vs DISO $(p=1.000)$, DIR vs DEXT $(p=1.000)$ and DISO vs DEXT $(p=1.000)$ comparisons there was no difference.

Regarding clinical relevance, it was observed that the difference between tasks 2 and 4 for TA presented a higher relevance $(d=0.87)$ and for the VMO, a moderate relevance $(d=0.55)$. The difference between tasks 2 and 3 showed high relevance for $S O(d=0.87)$, while that between tasks 3 and 4 presented high relevance for TA $(d=0.84)$, SO $(d=1.02)$ and VMO $(d=0.82)$. For the tasks ( 2 and $3 ; 3$ and 4$)$, there was a moderate relevance for GMX $(d=0.58)$ and GMED $(d=0.76)$ [9]. 
Table 1

Normalized EMG of muscle for each task.

TASKS

Mean(DP)

\begin{tabular}{|c|c|c|c|c|c|c|c|}
\hline \multirow{2}{*}{\multicolumn{2}{|c|}{$\begin{array}{l}\text { TASKS } \\
\text { Mean(DP) }\end{array}$}} & & & & \multirow{2}{*}{\multicolumn{3}{|c|}{ ANOVA }} \\
\hline & & & & & & & \\
\hline Muscles & $\mathbf{R}$ & DP & DIR & DISO & DEXT & $\mathbf{F}$ & $\mathrm{p}$ \\
\hline TA & $0.18(0.14)$ & $0.17(0.12)$ & $0.19(0.13)$ & $0.19(0.12)$ & $0.20(0.13)$ & 0.491 & 0.50 \\
\hline so & $0.22(0.15)$ & $0.37(0.25)$ & $0.57(0.27)$ & $0.54(0.24)$ & $0.56(0.24)$ & 20.618 & 0.001 * \\
\hline VMO & $0.38(0.28)$ & $0.43(0.32)$ & $0.38(0.28)$ & $0.39(0.26)$ & $0.42(0.29)$ & 0.457 & 0.767 \\
\hline RA & $0.25(0.11)$ & $0.24(0.10)$ & $0.25(0.11)$ & $0.25(0.11)$ & $0.25(0.11)$ & 0.745 & 0.567 \\
\hline TFL & $0.14(0.19)$ & $0.16(0.20)$ & $0.17(0.20)$ & $0.18(0.20)$ & $0.17(0.21)$ & 3.992 & $0.008^{*}$ \\
\hline GMX & $0.38(0.16)$ & $0.39(0.15)$ & $0.35(0.23)$ & $0.50(0.31)$ & $0.46(0.18)$ & 1.193 & 0.329 \\
\hline GMED & $0.40(0.30)$ & $0.47(0.35)$ & $0.52(0.45)$ & $0.55(0.47)$ & $0.54(0.40)$ & 2.547 & 0.054 \\
\hline$A D$ & $0.33(0.27)$ & $0.38(0.29)$ & $0.39(0.28)$ & $0.40(0.29)$ & $0.45(0.39)$ & 1.977 & 0.117 \\
\hline \multicolumn{8}{|c|}{$\begin{array}{l}\text { TA: tibialis anterior; SO: soleus; VMO: vastus medial oblique; RA: rectus abdominis; TFL: tensor } \\
\text { fasciae latae; GMX: gluteus maximus; GMED: gluteus medius; AD: adductor longus; R: rest; DP: } \\
\text { diagonal primitive; DIR: diagonal against isotonic resistance; DISO: diagonal against maximum } \\
\text { isometric resistance; DEXT: diagonal of the left upper limb and active extension of the right lower } \\
\text { limb; *Statistically significant. }\end{array}$} \\
\hline
\end{tabular}

\section{Discussion}

This study aimed to investigate the occurrence and the condition of the overflow from upper limb migrating to inferior contralateral supported limb in standing position. Our first hypothesis was confirmed just for the SO muscle although we found a clinical relevance for the other muscles. The higher EMG activity for SO muscle was in the following order. First, the DIR task (with isotonic contraction), followed by DEXT task (free diagonal, but with an extension of the right lower limb), then the DISO task (an isometric diagonal), followed by DP task (free diagonal), and finally, the lower EMG activity was in R task (just a proposed posture without diagonals, but maintained weight support). So, the second hypothesis was also confirmed because the muscle recruitment was superior in PNF diagonals (with the use of resistance) compared to free voluntary movement, but with differences between the muscles.

The results confirm the overflow of muscle activation from the upper limb to the contralateral supported lower limb. That occurrence was mainly during the DIR task (isotonic diagonal) as the condition with a more significant impact on the increase in muscle activity. The use of isometric diagonal is widespread to promote overflow principle (Gardner, 1963; Moore, 1975; Pink, 1981; Gontijo et al., 2012), but the performance of isotonic PNF diagonals can also change muscular activity in distant regions (Pink, 1981; Gontijo et al., 2012; Abreu et al., 2015; Nakada et al., 2018). The present results revealed the importance of the upper limb diagonal (flexion, abduction, and external rotation of the shoulder, an extension of 
elbow, wrist, and fingers) in the activity of soleus in standing position. We believe this position, in particular, needs stability, which can be acquired by the facilitation of soleus by the overflow. One crucial function of soleus is the contribution to a prolonged biped posture [14] Baudry, Penzer, \& Duchateau, 2014) in a tonic way. In this line of thought, we can assume that phasic activity, provided during the isotonic diagonal in the upper limb, modified muscle activation in a distant muscle with a predominant tonic activity. An important impact of these findings regards to the rehabilitation field, in the retraining of standing position and balance.

Although the other muscles did not show statistical differences, the moderate and high clinical relevance indicates they participated in overflow. Looking at under kinesiology viewpoint, we can say that all muscles contributed to joint stability to support the movement on the left upper limb. To elevate the upper limb above to head (diagonal primitive), it is necessary trunk extension, and that extension needs the trunk and pelvis stability. While the upper limb moves, the stability was provided by gluteus muscles, AD, RA, and TFL muscles [16]. The GMAX help to hip extension and the GMED and AD muscles to maintain the hip in a neutral position, followed by TFL, while the RA helped to keep the trunk and pelvis stability. Muscle activation also was necessary to achieve body stability during movement. First, SO activation produced plantar flexion, and foot moved against the ground. However, the TA activation produced a balance of SO activation and as a result, the ankle stability [17]. Finally, the VMO was activated to avoid increased flexion of the knee, maintained close to an extension [17].

The results of this study have a clinical application since many patients cannot stay standing in initial phases of recuperation of an orthopedic, traumatic, or neurological condition. The use of the present approach could improve the condition of distal muscles, mainly soleus, facilitating the acquisition of a standing position as soon as possible. Some examples of situations that could benefit from this approach can be postoperative surgery of knee, ankle when the load is released and cases of stroke or traumatic brain injury in acute or subacute phases.

It is possible to observe several limitations in this study. First, the small sample size does not allow us to generalize the results, but indicates the importance of soleus activity, after overflow intervention. Second, there was no study of kinematic and kinetic variables, which would provide a better understanding of the effects of overflow. It can be indicative of future studies. However, a first step, exploring EMG activity, gives us a start in the knowledge of the overflow effects in an important muscle for the stand position.

\section{Conclusion}

The results suggest that there is an overflow of the muscles from upper limb migrating to inferior contralateral supported limb muscles in a standing position, especially during diagonal against isotonic resistance. The approach used in this study could improve the condition of distal muscles, mainly soleus, facilitating the acquisition of a standing position as soon as possible.

\section{Declarations}




\section{Ethics approval and consent to participate}

All participants signed to inform consent approved by the Research Ethics Committee of the Federal University of Triângulo Mineiro, Brazil (no. 1647/2010).

\section{Consent for publication}

Not applicable.

\section{Availability of data and materials}

Not applicable.

\section{Competing interests}

The authors declare that they have no competing interests.

\section{Funding}

No funding was received for this study.

\section{Authors' contributions}

LAPSS, CRJAB and VCD contributed to conception, design, analysis, interpretation of data and drafting the manuscript. MBC carried out the data collection and involved in interpretation of data and drafting the manuscript. All authors read and approved the final manuscript.

\section{Acknowledgements}

The authors wish to thank all volunteers involved in this study and Gil Lucio Almeida, PhD for support of the laboratory equipment.

\section{List Of Abbreviations}

PNF: proprioceptive neuromuscular facilitation

R: rest

DP: diagonal primitive 
DIR: diagonal against isotonic resistance

DISO: diagonal against maximum isometric resistance

DEXT: diagonal of the left upper limb and, concomitantly, perform an active extension of the right lower limb that was flexed at $15^{\circ}$.

TA: tibialis anterior

SO: soleus

VMO: vastus medial oblique

RA: rectus abdominis

TFL: tensor fasciae latae

GMX: gluteus maximus

GMED: gluteus medius

$A D$ : adductor longus

\section{References}

1. Hwang IS, Abraham LD. Quantitative EMG analysis to investigate synergistic coactivation of ankle and knee muscles during isokinetic ankle movement. Part 2: Time frequency analysis. J Electromyogr Kinesiol. 2001;11:327-35.

2. Adamson M, MacQuaide N, Helgerud J, Hoff J, Kemi OJ. Unilateral arm strength training improves contralateral peak force and rate of force development. Eur J Appl Physiol. Springer; 2008;103:5539.

3. Gontijo LB, Pereira PD, Neves CDC, Santos AP, Machado D de CD, Bastos VH do V. Evaluation of Strength and Irradiated Movement Pattern Resulting from Trunk Motions of the Proprioceptive Neuromuscular Facilitation. Rehabil Res Pract [Internet]. 2012 [cited 2020 Apr 9];2012:1-6. Available from: https://www.hindawi.com/journals/rerp/2012/281937/

4. Reznik JE, Biros E, Bartur G. An electromyographic investigation of the pattern of overflow facilitated by manual resistive proprioceptive neuromuscular facilitation in young healthy individuals: $A$ preliminary study. Physiother Theory Pract [Internet]. Taylor and Francis Ltd; 2015 [cited 2020 Apr 9];31:582-6. Available from: http://www.ncbi.nlm.nih.gov/pubmed/26452149

5. Abreu R, Lopes AA, Sousa ASP, Pereira S, Castro MP. Force irradiation effects during upper limb diagonal exercises on contralateral muscle activation. J Electromyogr Kinesiol [Internet]. Elsevier Ltd; 2015 [cited 2020 Apr 9];25:292-7. Available from: http://www.ncbi.nlm.nih.gov/pubmed/25592384 
6. Souza L, Baptista C, Brunelli F, Dionisio V. Effect and length of the overflow principle in proprioceptive neuromuscular facilitation: electromyographic evidences. Int J Ther Rehabil Res [Internet]. 2014;3:6. Available from: http://www.scopemed.org/fulltextpdf.php?mno=154883

7. Adler S, Beckers D, Buck M. Facilitação Neuromuscular Proprioceptiva. São Paulo; 1999.

8. Hermens HJ, Freriks B, Disselhorst-Klug C, Rau G. Development of recommendations for SEMG sensors and sensor placement procedures. J Electromyogr Kinesiol [Internet]. 2000 [cited 2018 Mar 10];10:361-74. Available from: http://www.ncbi.nlm.nih.gov/pubmed/11018445

9. Cohen J. Statistical Power Analysis for the Behavioral Sciences. 2nd ed. New York: Lawrence Erlbaum Associates; 1988.

10. Gardner GW. Specificity of strength changes of the exercised and nonexercised limb following isometric training. Res. Q. Am. Assoc. Heal. Phys. Educ. Recreat. Taylor \& Francis Group ; 1963. p. 98-101.

11. Moore JC. Excitation overflow: an electromyographic investigation. Arch Phys Med Rehabil [Internet]. 1975 [cited 2020 Apr 9];56:115-20. Available from: http://www.ncbi.nlm.nih.gov/pubmed/1119917

12. Pink M. Contralateral effects of upper extremity proprioceptive neuromuscular facilitation patterns. Phys Ther. 1981;61:1158-62.

13. Nakada CS, Meningroni PC, Ferreira ACS, Hata L, Fuzaro AC, Júnior WM, et al. Ipsilateral proprioceptive neuromuscular facilitation patterns improve overflow and reduce foot drop in patients with demyelinating polyneuropathy. J Exerc Rehabil [Internet]. Korean Society of Exercise Rehabilitation; 2018 [cited 2020 Apr 9];14:503-8. Available from: http://www.ncbi.nlm.nih.gov/pubmed/30018940

14. Lamm BM, Paley D, Herzenberg JE. Gastrocnemius soleus recession: A simpler, more limited approach [Internet]. J. Am. Podiatr. Med. Assoc. American Podiatric Medical Association; 2005 [cited 2020 Apr 9]. p. 18-25. Available from: http://www.ncbi.nlm.nih.gov/pubmed/15659410

15. Baudry S, Penzer F, Duchateau J. Input-output characteristics of soleus homonymous la afferents and corticospinal pathways during upright standing differ between young and elderly adults. Acta Physiol [Internet]. 2014 [cited 2020 Apr 9];210:667-77. Available from: http://www.ncbi.nlm.nih.gov/pubmed/24433254

16. Ekstrom R a, Donatelli R a, Carp KC. Electromyographic analysis of core trunk, hip, and thigh muscles during 9 rehabilitation exercises. J Orthop Sports Phys Ther [Internet]. 2007 [cited 2012 Nov 10];37:754-62. Available from: http://www.ncbi.nlm.nih.gov/pubmed/18560185

17. Dionisio VC, Almeida GL, Duarte M, Hirata RP. Kinematic, kinetic and EMG patterns during downward squatting. J Electromyogr Kinesiol [Internet]. 2008 [cited 2012 Nov 1];18:134-43. Available from: http://www.ncbi.nlm.nih.gov/pubmed/17029862 